\title{
A Comparative Study to Evaluate Selective COX-2 Inhibitors Alone or in Combination with Pregabalin Given Pre-Operatively For Pain Management in Total Knee Arthroplasty.
}

\author{
Dr. Ganaparthi Kamalahassan ${ }^{1}$,Dr.A.V.Gurava Reddy ${ }^{2}$,Dr. Krishna Kiran \\ Eachempati ${ }^{3}$,Dr. Sukesh Rao Sankineani ${ }^{4}$,Dr. Saketh Kolla ${ }^{5}$ \\ 1, 2, 3, 4,5 (Department Of Arthroplasty, Sunshine Hospitals, Secunderabad, Telangana State, India)
}

\begin{abstract}
Pain control during immediate post operative period is necessary in Total Knee Arthroplasty(TKA) patients for early mobilization. The use of COX-2 inhibitors preoperatively has shown significant reduction in opiod usage and in pain relief post operatively. Furthermore, some studies have suggested that the addition of pregabalin preoperatively has led to further reduction in postoperative pain and opiod use.The aim of this study is to compare pain scores in a group of patients who receive a single preoperative dose of pregabalin combined with a COX-2 inhibitor and a control group which receives only COX-2 inhibitor. A prospective randomiazed control study was carried in which 200 patients undergoing primary unilateral total knee replacement received preoperatively either $200 \mathrm{mg}$ celecoxib alone (Group A, $n=100$ ) or combination of celecoxib $200 \mathrm{mg}$ plus $75 \mathrm{mg}$ of Pregabalin (Group B $n=100$ ) approximately one hour before the surgery. All patients underwent primary unilateral TKA under spinal analgesia using the standard medial parapatellar approach under tourniquet. Pain was assessed pre operatively, after 6 hours, 24 hours, 48 hours post operatively using Visual Analogue scale $(V A S)$ in all patients.Both the groups were comparable regarding the demographic parameters and showed a significant postoperative pain relief at the end of 48 hours $(p<0.05)$. The mean preoperative VAS score in group $A$ was 5.82 which decreased to 2.86 after 48 hours ( $p<0.05)$. Similarly, mean preoperative VAS score in group $B$ was 5.12 which decreased to 2.05 after 48 hours after surgery. No significant intergroup difference in functional outcome was noted. However, mean cumulative opioid consumption was lower in group B compared to group A $(p<0.05)$.Preoperative administration of celecoxib lowers the pain scores in the post operative period. Addition of pregabalin reduces the postoperative pain significantly in TKA patients.
\end{abstract}

Keywords: selective COX-2 inhibitors, pregabalin, pain management, VAS score, TKA.

\section{Introduction}

Pain management during immediate post operative period following Total Knee Arthroplasty(TKA) is utmost priority for every surgeon for patient comfort, satisfaction and early mobilization ${ }^{1}$. Despite the developments in management of pain modalities more than 50\% of patients undergoing TKA experience varying degrees of pain. Severe degree of pain not only has negative influence on recovery but can also result in catastrophic events like myocardial ischaemia and several organ dysfunctions, therefore effective postoperative pain management is a key step in successful TKA. Most important concept of current pain management is multimodal approach. "Preemptive" refers to initiate pain management before surgical stimuli, in addition to multimodal approach which means use of more than two drugs or modalities with different mechanism of action having synergistic effects, both of which has been used in our study.

\section{Aim Of Study}

To compare pregabalin in combination with COX-2 inhibitor vs COX -2 inhibitor alone given in patients prior to TKA in terms of postoperative pain relief.

\section{Methodology}

It was a prospective study. Ethical clearance was taken from our institution comitte. 200 patients fulfilling inclusion criteria were selected with written consent. Celecoxib Intolerance, Class III/IV congestive Heart Failure, Myocardial Infarction, GI Bleed,NSAID Intolerance ,Renal Insufficiency (Serum Creatinine > 1.2 $\mathrm{mg} / \mathrm{dL}$ ),coagulation disorder, patients younger than 45 or older than 85 years, liver failure patients, Adductor canal block, Femoral canal block, Staggered TKR, prior pregabalin and COX-2 inhibitors usage were excluded from the study. Group A included 100 patients who received 2 drugs orally - 200mg of celecoxib and $75 \mathrm{mg}$ of pregabalin 1 hour prior to surgery. Group B included 100 patients who were given single dose of $200 \mathrm{mg}$ of celecoxib 1 hour prior to Total Knee Arthroplasty. All patients underwent primary unilateral TKA under spinal analgesia using the standard medial parapatellar approach under tournique. Pain was assessed pre operatively and 6,24,48 hours post operatively using Visual Analogue scale (VAS). Visual analogue pain scores ${ }^{15}$ were 
converted to a standardized 0 to 10 scale with 0 indicating no pain and 10 indicating worst possible pain to avoid subjective errors of assessment.

\section{Results}

\begin{tabular}{|l|l|l|}
\hline & Group A & Group B \\
\hline Mean Age & 62.92 & 62.90 \\
\hline Male/Female & $28 / 72$ & $19 / 81$ \\
\hline
\end{tabular}

Mean age of patients in Group A and B was 62.92 and 62.90 yrs which was comparable. $72 \%$ were females and $28 \%$ males in Group A as compared to $81 \%$ females and $19 \%$ males in Group B, which showed a higher incidence of joint pathology in females.

Difference In Pain Scores
Comparison within the group:
\begin{tabular}{|l|l|l|l|l|l|l|}
\hline \multirow{3}{*}{ Time } & VISUAL ASSESMENT PAIN SCORE \\
\cline { 2 - 7 } & Group-A & \multicolumn{4}{l|}{ Group-B } \\
\cline { 2 - 7 } & Mean & Median & SD & Mean & Median & SD \\
\hline Pre operative & 5.2 & 5 & 3 & 5.9 & 5 & 3 \\
\hline 6 Hrs post op & 3.9 & 3 & 3 & 5.2 & 5 & 3 \\
\hline 24 Hrs post op & 3.0 & 2 & 2 & 3.9 & 4 & 2 \\
\hline 48 Hrs post op & 2.1 & 1 & 2 & 2.5 & 2 & 2 \\
\hline P-value & $<0.01(\mathrm{HS})$ & & $<0.01(\mathrm{HS})$ & \\
\hline Pre-op vs 6 Hrs & $<0.01(\mathrm{HS})$ & \multicolumn{2}{l|}{$0.046(\mathrm{~S})$} \\
\hline 6Hrs vs 24Hrs & $<0.01(\mathrm{HS})$ & $<0.01(\mathrm{HS})$ \\
\hline 24Hrs vs 48Hrs & $<0.01(\mathrm{HS})$ & $<0.01(\mathrm{HS})$ \\
\hline
\end{tabular}

(S-Significant,HS-Highly significant,NS-Not significant)

Using Wilcoxon test pain scores were compared between two time intervals and was observed that there was significant reduction in pain scores in both the groups when preoperative pain scores were compared with post operative scores and significant pain reduction was seen at 24 and 48 hours.

\section{Comparison between the groups:}

\begin{tabular}{|l|l|l|l|l|l|l|l|}
\hline \multirow{2}{*}{ Difference } & \multicolumn{2}{|l|}{ Group-A } & \multicolumn{2}{l|}{ Group-B } & \multirow{2}{*}{ P-value } \\
\cline { 2 - 8 } & Mean & Median & SD & Mean & Median & SD & \\
\hline At6rs to Pre OP & -1.3 & -2 & 3 & -0.7 & -1 & 3 & $0.13(\mathrm{NS})$ \\
\hline At24rs to At6Hrs & -0.9 & -1 & 2 & -1.2 & -1 & 3 & $0.52(\mathrm{NS})$ \\
\hline At48rs to At24Hrs & -1.0 & -1 & 2 & -1.5 & -1 & 2 & $<0.01(\mathrm{HS})$ \\
\hline
\end{tabular}

Statistical analysis was performed using Mann-Whitney U test. It was observed that there was no statistically significant difference among both groups in terms of pain relief at 6 and 24 hours but a significant pain reduction was seen at 48 hours in group $\mathrm{A}$ as compared to group $\mathrm{B}(\mathrm{P}$ value $<0.01)$.

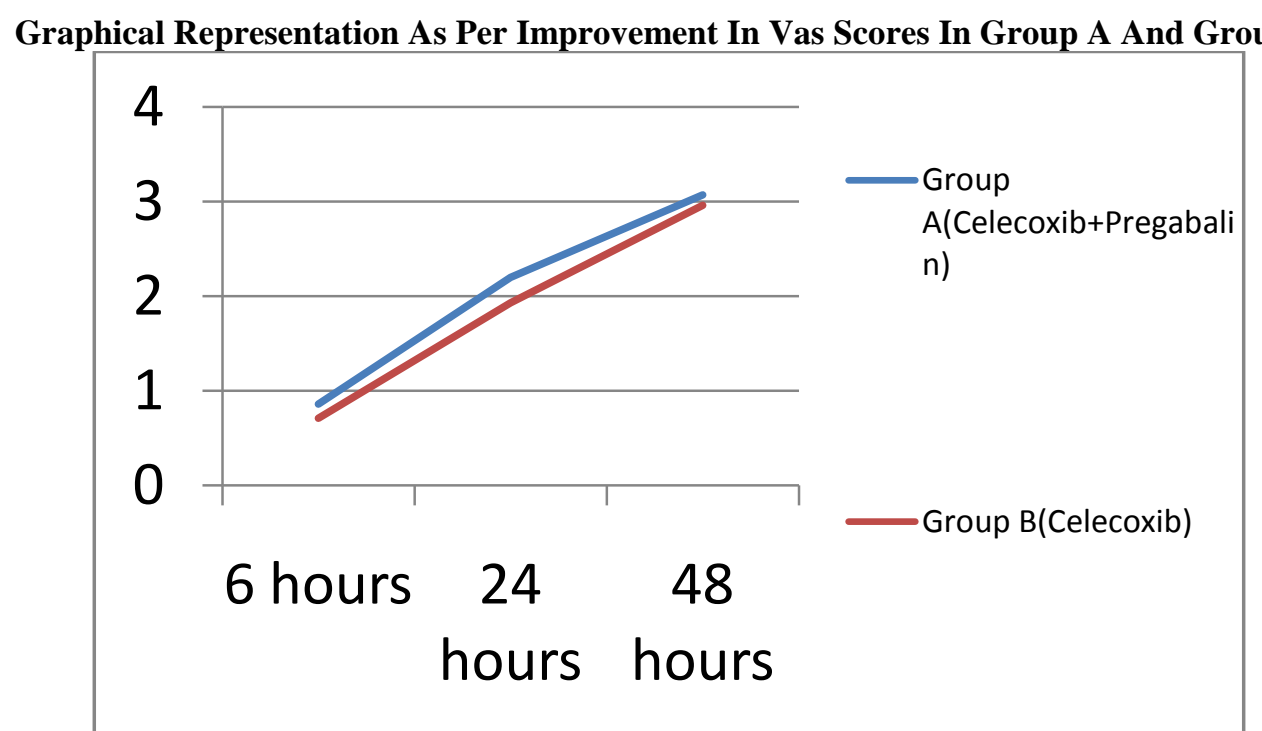




\section{Discussion}

In our study there was significant reduction of pain score in group A and B postoperatively when compared to pre op scores. Though 48 hours pain relief was significantly reduced not much statistically significant difference was observed with Group A at 6 and 24 hours as compared to group B.

Asokumar Buvanendran, S. Korian et $\mathrm{al}^{2}$ studied effect of pregabalin and celecoxib administration perioperatively and observed significant pain reduction and lower requirements of opiod usage postoperatively,yet they used higher doses of pregabalin $(300 \mathrm{mg})$ which was associated with side effects like dizziness and insomnia. In our study there were no significant side effects in either group because lower dose of pregablin(75mg) was used.

Kenneth J. Tuman observed Inadequate pain management after TKA results in delayed rehabilitation and poor functional recovery ${ }^{3}$. Pain control is correlated with patient satisfaction ${ }^{4-6}$. Hence, multimodal pain management protocols are being used widely which block different pain pathways ${ }^{7,8}$.NSAIDs are mainstay and preoperative use of selective COX-2 inhibitors for TKA reduces opiod consumption ${ }^{9}$ post operatively. Pregabalin is an anti-neuropathic drug known to have a role in post operative pain management by reducing central sensitization ${ }^{10}$. No significant side effects were observed with celecoxib or pregabalin usage.

Buvanendran et $\mathrm{al}^{11}$ found significant reduction of pain after using pregabalin $300 \mathrm{mg}$ orally before and for two weeks following TKA,however higher dose of pregabalin is associated with systemic side effects. Lower dose can prevent these side effects.

\section{Conclusion}

Guidelines for pain management in TKA patient suggest use of preemptive and multimodal method for management of pain .[level of evidence-1,grade A recommendation $]^{12}$.Use of nonselective COX inhibitors can result in intraoperative bleeding therefore use of selective cox-2 inhibitors help in better management. Synergistic benefits of Pregabalin and celecoxib are seen up to 72hrs.

Preoperative administration of Celecoxib lowers the pain scores in the post operative period but addition of Pregabalin reduces the post operative pain significantly,therefore combination therapy can be used for better pain management in TKA post operatively which is both patients and surgeon concern.

\section{References}

[1]. Borgeat A.The role of regional anaesthesia in patient outcome : orthopaedic surgery.Tech Reg Anesth Pain Manag 2008;12:178.

[2]. Asokumar Buvanendran,Jeffrey S. Kroin,et al.Perioperative Oral Pregabalin Reduces Chronic Pain After Total Knee Arthroplasty: A Prospective, Randomized, Controlled Trial. Pain Medicine Vol. 110, No. 1, January 2010 (199)

[3]. Capdevila X, Barthelet Y, Biboulet P, et al. Effects of perioperative analgesic technique on the surgical outcome and duration of rehabilitation after major knee surgery. Anaesthesiology 1999;91:8.

[4]. Parvizi J,Miller AG and Gandhi K.Multimodal pain management after total joint arthroplasty.J Bone Joint Surgggg Am 93:10751084.

[5]. Brokelman RB,van Loon CJ, Rijnbergg WJ. Patient versus surgeon satisfaction after total hip arthroplasty. J Bone Joint Surg Br 2003;85:495.

[6]. Wall PD. The prevention of post operative pain. Pain 1988;33:289.

[7]. Dorr LD, Raya J, Long WT, et al. Multimodal analgesia without parenteral narcotics for total knee arthroplasty. J Arthroplasty 2008;23:502.

[8]. Lavernia C, Cardona D, Rossi MD, et al. Multimodal pain management and arthrofibrosis. J Arthroplasty 2008;23(6 Suppl 1):74.

[9]. Straube S, Derry S, McQuay HJ, et al. Effect of preoperative Cox-II-selective NSAIDs(coxibs) on post operative outcomes: a systematic review of randomized studies. Acta Anaesthesiol Scand 2005;49:601.

[10]. Ho KY,Gan TJ. Habib AS. Gabapantin and post operative pain- a systematic review of randomized controlled trials. Pain 2006;126:91.

[11]. Buvanendran A, Kroin JS, Della ValleCJ, et al. Perioperative oral pregabalin reduces chronic pain after total knee arthroplasty : a prospective, randomized, controlled trial. Anesth Analg 2010;110:199.

[12]. Korean Knee Society.Guidelines for the Management of Postoperative Pain after Total Knee Arthroplasty.Knee Surg Relat Res. 2012 Dec; 24(4): 201-207. Published online 2012 Nov 29. doi: 10.5792/ksrr.2012.24.4.201 